\title{
Cardiac metastasis from a renal cell carcinoma
}

\author{
Abdulaziz Alghamdi MD¹, James Tam MD²
}

\begin{abstract}
A Alghamdi, J Tam. Cardiac metastasis from a renal cell carcinoma. Can J Cardiol 2006;22(14):1231-1232.
\end{abstract}

A 59-year-old man developed an episode of syncope while he was driving. This resulted in a motor vehicle accident, and the patient sustained an open fracture of the left femur. Biopsy of the left femur fracture showed a metastastic renal cell carcinoma, and echocardiography revealed a right ventricular mass without contiguous vena caval or right atrial involvement. This is one of the few reported cases of renal cell carcinoma associated with syncope as an initial symptom.

\section{Néphrocarcinome associé à une lésion métastastique au cœur}

Un homme de 59 ans a fait une syncope au volant; il s'en est suivi un accident de la route et le conducteur a subi une fracture ouverte du fémur gauche. Une biopsie du fémur en question a révélé la présence d'un néphrocarcinome métastatique, et l'échocardiographie, une masse dans le ventricule droit, sans atteinte contiguë de la veine cave ou de l'oreillette droite. Il s'agit là de l'un des rares cas signalés de néphrocarcinome associé à une syncope comme premier symptôme.

Key Words: Cardiac metastasis; Renal cell carcinoma; Syncope

\section{CASE PRESENTATION}

A 59-year-old man with a history of well-controlled hypothyroidism experienced sudden-onset palpitations and syncope while he was driving. The patient lost consciousness and, as a result, was involved in a motor vehicle accident. On arrival to the emergency room, clinical and radiographic examination showed an open fracture of the left femur. The patient had no history of antecedent chest pain, shortness of breath or dizziness.

On physical examination, the patient's vital signs were stable (blood pressure $138 / 80 \mathrm{mmHg}$ and heart rate 92 beats/min) and his jugular venous pressure was not distended. The patient's lungs were clear on auscultation with no abnormalities in the cardiac examination. The patient's abdomen was soft and nontender. An obvious open fracture of the left femur was diagnosed.

An electrocardiogram showed normal sinus rhythm, with $\mathrm{T}$ wave abnormality and a prolonged QT interval (QT/QTc $392 \mathrm{~ms} / 495 \mathrm{~ms}$ ) (Figure 1). A cardiology consultation was requested preoperatively to assess the cause of the underlying syncope and determine the significance of the abnormal electrocardiogram finding. Cardiac arrhythmia was suspected, and therefore, echocardiography was suggested.

The patient was taken to the operating room for an open reduction and internal fixation of the left femur fracture as an emergency case. The patient underwent an uneventful and uncomplicated operation under general anesthesia. In the meantime, an intraoperative inspection of the fracture was performed on noticing an unknown mass. An incisional biopsy was carried out and the specimen was sent for histopathology. Postoperatively, transthoracic echocardiography showed marked asymmetrical left ventricular hypertrophy at the septum predominantly involving the right ventricular component of the interventricular septum. A small, noncompressive pericardial effusion was also present (Figure 2). There was no mass within the inferior vena cava or in the right atrium. Ultrasound of the abdomen showed a $13 \mathrm{~cm}$ left renal mass without renal obstruction. The histopathology of the mass biopsied from the left femur was consistent with metastatic renal cell carcinoma.

\section{DISCUSSION}

Tumours that involve the heart are more likely to represent metastatic disease than primary cardiac neoplasm (1). The prevalence of primary cardiac tumours among general autopsies is less than one in 3500 (2). The most common primary cardiac neoplasm is myxoma, usually arising from the left atrium, and other rare benign tumours include fibromas, lipomas and rhabdomyomas. Others, such as mesotheliomas, angiosarcomas and rhabdomyosarcoma, are examples of malignant primary cardiac tumours.

There are three general mechanisms of cardiac involvement: direct extrinsic compression by tumour mass, diffuse intramyocardiac infiltration and intracameral extension either as a consequence of local tumour growth or venous extension $(3,4)$. Lung and breast cancer are considered to be the most common causes of cardiac metastases.

Approximately $45 \%$ of patients with renal carcinoma present with localized tumours, $25 \%$ of patients present with locally advanced disease, and approximately $30 \%$ of patients may have metastases at the time of diagnosis. The most common metastastic sites are the lungs, bone, soft tissues, liver and central nervous system.

Hypertension is the most common cardiac presentation of renal cell carcinoma and occurs in $20 \%$ to $37.5 \%$ of patients. Other cardiac presentations include shortness of breath, cough, arrhythmia, chest pain and peripheral edema.

${ }^{1}$ Department of Anesthesia, College of Medicine, University of Manitoba; ${ }^{2}$ Department of Cardiology, Health Sciences Centre, Winnipeg, Manitoba

Correspondence: Dr Abdulaziz Alghamdi, Department of Anesthesia, College of Medicine, University of Manitoba, Room LB,

315 Lennox Bell Lodge, 60 Pearl Street, Winnipeg, Manitoba R3E 1X2. Telephone 204-787-1798, fax 204-787-4291, e-mail mosare@yahoo.com Received for publication March 31, 2005. Accepted November 3, 2005 


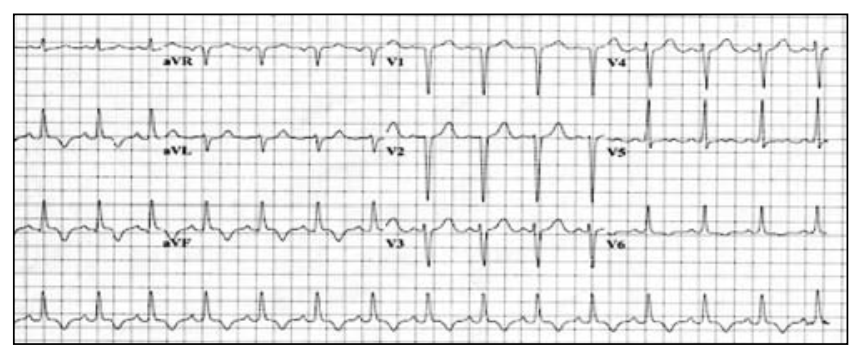

Figure 1) Electrocardiogram showing normal sinus rhythm, with $T$ wave abnormality and prolonged QT interval (QT/QTc 392 ms/495 ms)

Syncope is most often due to vasovagal reactions. Other causes include tachydysrhythmias, heart block, obstruction of left ventricular outflow and orthostatic hypotension. Syncope must be differentiated from seizures and other states of altered consciousness. It is estimated that syncope accounts for $1 \%$ to $6 \%$ of hospital admissions and 3\% of emergency room visits.

In the present case report, the patient had renal cell carcinoma with proven metastatic spread to the femur, as confirmed histopathologically. Echocardiography suggested cardiac involvement of the right ventricular wall.

On reviewing the literature, the present case report is one of the few cases that describes a solitary, right ventricular, metastatic tumour arising from renal cell carcinoma. Although no formal electrophysiological testing was performed, we suspect that a tachyarrhythmia may have led to syncope and the subsequent motor vehicle accident. The case also emphasizes the importance of a thorough history review and physical examination in the work-up of patients with syncope.

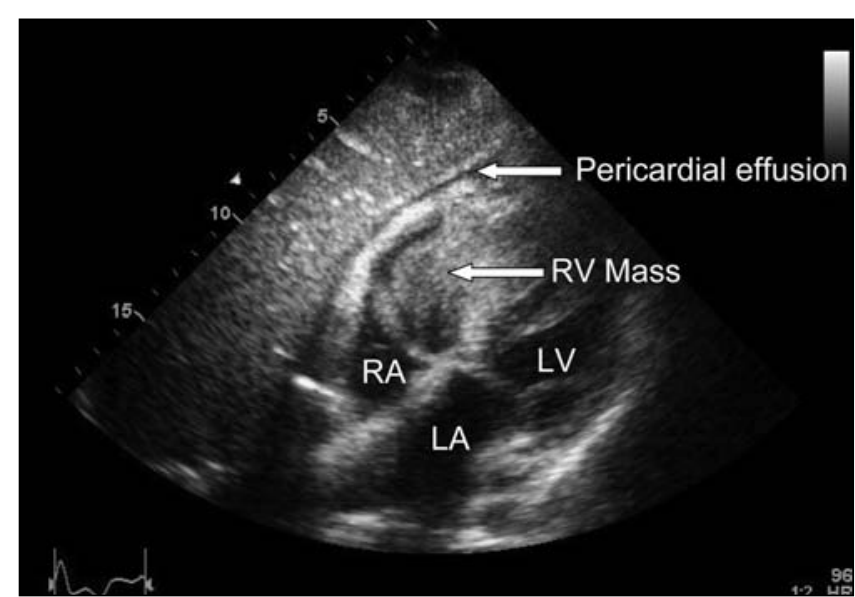

Figure 2) Subcostal four-chambered view demonstrating marked thickening of the right interventricular septum with a tiny, right ventricular cavity and minimal pericardial effusion. LA Left atrium; LV Left ventricle; RA Right atrium; RV Right ventricle

\section{REFERENCES}

1. Lam KY, Dickens P, Chan AC. Tumors of the heart. A 20-year experience with a review of 12,485 consecutive autopsies. Arch Pathol Lab Med 1993;117:1027-31.

2. Roberts WC. Primary and secondary neoplasms of the heart. Am J Cardiol 1997;80:671-82.

3. Mukai K, Shinkai T, Tominaga K, Shimosato Y. The incidence of secondary tumors of the heart and pericardium: A 10-year study. Jpn J Clin Oncol 1988;18:195-201.

4. Nakayama R, Yoneyama T, Takatani O, Kimura K. A study of metastatic tumors to the heart, pericardium and great vessels. I. Incidences of metastases to the heart, pericardium and great vessels. Jpn Heart J 1966;7:227-34. 